Article

\title{
Evolutionarily Distant Streptophyta Respond Differently to Genotoxic Stress
}

\author{
Radka Vágnerová ${ }^{1}$, Alena Lukešová ${ }^{2}$, Martin Lukeš $^{3}$, Petra Rožnovská ${ }^{1}$, Marcela Holá $^{1}$, \\ Jana Fulnečková ${ }^{4}$, Jiří Fajkus 5,6 (iD and Karel J. Angelis 1,* \\ 1 Institute of Experimental Botany, Czech Academy of Sciences, v.v.i., Na Karlovce 1, 16000 Prague 6, \\ Czech Republic; radka.vagnerova@centrum.cz (R.V.); roznovska@gmail.com (P.R.); \\ marcelahola@seznam.cz (M.H.) \\ 2 Biology Centre ASCR, v.v.i., Institute of Soil Biology, Na Sádkach 7, 37005 České Budějovice, Czech Republic; \\ luksa@upb.cas.cz \\ 3 Institute of Microbiology ASCR, Centrum Algatech, Laboratory of photosynthesis, Opatovický mlýn, \\ 37981 Třeboň, Czech Republic; lukesm@alga.cz \\ 4 Institute of Biophysics, Czech Academy of Sciences, v.v.i., Královopolská 135, 61265 Brno, Czech Republic; \\ fulneckova@ibp.cz \\ 5 Laboratory of Functional Genomics and Proteomics, NCBR, Faculty of Science, Masaryk University, \\ Kotlářská 2, 61137 Brno, Czech Republic; fajkus@sci.muni.cz \\ 6 Mendel Centre for Plant Genomics and Proteomics, CEITEC-Central European Institute of Technology, \\ Masaryk University, Kamenice 5, 62500 Brno, Czech Republic \\ * Correspondence: karel.angelis@gmail.com; Tel.: +420-224-322-603
}

Received: 31 August 2017; Accepted: 14 November 2017; Published: 17 November 2017

\begin{abstract}
Research in algae usually focuses on the description and characterization of morpho-and phenotype as a result of adaptation to a particular habitat and its conditions. To better understand the evolution of lineages we characterized responses of filamentous streptophyte green algae of the genera Klebsormidium and Zygnema, and of a land plant—-the moss Physcomitrella patens - to genotoxic stress that might be relevant to their environment. We studied the induction and repair of DNA double strand breaks (DSBs) elicited by the radiomimetic drug bleomycin, DNA single strand breaks (SSB) as consequence of base modification by the alkylation agent methyl methanesulfonate (MMS) and of ultra violet (UV)-induced photo-dimers, because the mode of action of these three genotoxic agents is well understood. We show that the Klebsormidium and Physcomitrella are similarly sensitive to introduced DNA lesions and have similar rates of DSBs repair. In contrast, less DNA damage and higher repair rate of DSBs was detected in Zygnema, suggesting different mechanisms of maintaining genome integrity in response to genotoxic stress. Nevertheless, contrary to fewer detected lesions is Zygnema more sensitive to genotoxic treatment than Klebsormidium and Physcomitrella
\end{abstract}

Keywords: Physcomitrella patens; Klebsormidium; Zygnema; DNA damage and repair; bleomycin; methyl methanesulfonate; ultraviolet light

\section{Introduction}

Responses of living organisms to DNA damage caused by either intrinsic or environmental factors must act promptly and with a high efficiency to preserve integrity and functionality of genetic information. Although algae play an important ecological role and are considered strong indicators of environmental changes, besides ultra violet light (UV), only few studies have investigated their responses to genotoxic stress and DNA damage. To our knowledge, there is only one report on the response of an Arctic alga Zygnema to ionizing radiation (IR) [1]. This is why the purpose of this study is to identify and describe differences in sensitivity to induction and response to genotoxic exposure of 
algae species from two genera-Klebsormidium and Zygnema - as well as moss Physcomitrella patens as representatives of Streptophyta lineages.

The Streptophyta studied have filamentous morphology, are widespread all over the world and belong to ecologically important organisms, often forming mats or crusts. Algae of both genera live in environments where they have to cope with severe conditions, such as Zygnema species occupying hydro-terrestrial habitats [2-4] and Klebsormidium species inhabiting aero-terrestrial habitats [5-7]. For comparison of genotoxic sensitivity, we used in parallel to algae moss, a bryophyte, P. patens that is recognized as an elegant model of not flowering plants [8]. Bryophytes represent the oldest living branch in land plant evolution [9] and moss protonemata morphologically resemble algal filaments.

Species that successfully transitioned from aqueous to terrestrial environments acquired a phenotype adapted to the new conditions. To characterize differences in sensitivity phenotype among Klebsormidium, Zygnema and Physcomitrella, we took advantage of their morphology and studied their sensitivity to genotoxic treatment in cultures of short filament fragments. The use of cultures with enriched fraction of dividing cells was previously established in the moss P. patens [10-12]. The clonal propagation of these cells amplifies the potential effects and consequences of incurred DNA damage to plant growth and phenotype.

To identify differences among studied Streptophyta, we studied their sensitivity and response to three types of genotoxic attacks, predicted as relevant to the conditions of their environment. Firstly, oxidative stress exerted by bleomycin, which mimics the impact of IR by generating a burst of reactive oxygen species (ROS), which interact with DNA and are responsible for induction of solitary as well as clustered DNA lesions [13]. Burst of ROS in plants also accompanies other biotic and abiotic stresses including desiccation or salinity. Secondly, we studied effects of methyl methanesulfonate (MMS), a $\mathrm{S}_{2} \mathrm{~N}$ alkylation agent, which attacks DNA bases as an alkylation modifier like nitrosoureas, and similarly naturally occurring nitrosocompounds. Thirdly, as an example of genotoxic physical stress, we used UVC to induce cyclobutene pyrimidine (CPD) and $6^{\prime}-4^{\prime}$ pyrimidine-pyromidone (6-4PP) photo-dimers. UVC was used instead of UVB to simulate terrestrial solar UV exposure; because the yield and ratio of UVC and UVB induced photo-dimers is identical and the use of UVC is experimentally simpler. In addition, both UVC and UVB radiation are reported to interfere with photosynthesis and thus to attenuate the growth of algal cells [14].

\section{Materials and Methods}

\subsection{Plant Material}

Algae-Six strains from orders Klebsormidiales and Zygnematales were studied. Five axenic strains K101-Klebsormidium flaccidum SAG 7.91, K 292-Klebsormidium elegans SAG 7.96, K293-K. flaccidum SAG 2307, Z181-Zygnema circumcarinatum SAG 698-1aZ294-Zygnema sp. SAG 698-4 were obtained from Sammlung von Algenkulturen (SAG), University of Goettingen, Germany. Strain Z436-Zygnema sp., freshly isolated from a shallow seepage pool in Petunia Bay, Svalbard (High Arctic) was kindly provided by Martina Pichrtova, FS, Charles University, Prague, Czech Republic.

Algae were grown on bold-basal medium (BBM) agar plates [15] at 20-24 $\mu \mathrm{mol}$ photons $\mathrm{m}^{-2} \mathrm{~s}^{-1}$



Moss-P. patens "Gransden 2004" wild type used in this study was kindly provided by Andrew C. Cuming, CPS, Univ. Leeds, UK. Physcomitrella was vegetatively propagated by weekly subculture of homogenized protonemata on routine basal BCD agar medium supplemented with $1 \mathrm{mM} \mathrm{CaCl}_{2}$ and $5 \mathrm{mM}$ ammonium tartrate as a biomass on Petri plates overlaid with cellophane in growth chambers with $18 / 6 \mathrm{~h}$ day/night cycle at $22 / 18^{\circ} \mathrm{C}$ [16].

\subsection{Algal Growth and Phenotype Assays}

Biomass of Klebsormidium K101 and Zygnema Z436 in exponential phase of growth were collected from Petri plates and gently sheared in fresh liquid BBM medium. Aliquots of suspension culture 
were then treated with indicated concentrations of bleomycin $(0-300 \mu \mathrm{g} / \mathrm{mL})$ and MMS $(0-200 \mathrm{mM})$ in BBM medium for $30 \mathrm{~min}$. After the treatment cultures were washed by 3 cycles of centrifugation and suspension in fresh medium and finally resuspended as a 5-times concentrate of the original volume. $15 \mu \mathrm{L}$ of untreated or treated suspension were spotted as inocula in sectors on agar plates and grown under continuous light at $25^{\circ} \mathrm{C}$ for 14 days. Five spots per concentration sector on plate and 6 replica plates were set for every experiment. Growth of spot inocula was monitored and photo-documented. For microscopy, the algae were cultivated as liquid culture in 24-well microtiter plates, $200 \mu \mathrm{L}$ of algal suspension of each treatment was transferred into a well and volume was adjusted to $1.5 \mathrm{~mL}$. There were 4 replicas of each treatment. 30 filaments per treatment were reviewed and photographed using Olympus BX 51 microscope equipped with Olympus DP 50 digital camera (Olympus Czech Group sro., Prague, Czech Republic) on the 1st, 7th and 14th day after the treatment. In total, 3 independent experiments were performed for both growth and cell damage monitoring.

\subsection{Genotoxin Treatments for DNA Damage Quantification}

Biomass of vegetatively propagated Klebsormidium, Zygnema and P. patens were collected from plates, suspended in $8 \mathrm{~mL}$ of BBM or BCD media per plate respectively and sheared with a T25 homogenizer (IKA, Staufen, Germany) at 10,000 rpm for two 1-min cycles. Sheared filament culture was left for overnight recovery in a cultivation chamber with gentle shaking at $100 \mathrm{rpm}$. This preparation yielded suspension cultures of $2-7$ cell filaments, which can be distributed by pipetting and collected by filtering through $50 \mu \mathrm{m}$ mesh Partec CellTrics filters (Sysmex Deutschland GmbH, Norderstedt bei Hamburg, Germany). Adopted from [11].

Bleomycin as Bleomedac inj. (Medac, Hamburg, Germany) and MMS cat. M4016 (Sigma-Aldrich sro., Prague, Czech Republic) were used for genotoxin treatments as freshly prepared solutions from weighed or pipetted reagent in BBM or BCD media, respectively. UVC treatment was carried out in a Hoeffer UVC 500 cross-linker at $254 \mathrm{~nm}$. Irradiation and the following steps until harvest and freezing of samples were performed in the dark or under red illumination to block photolyases, which are activated by blue light $(435 \mathrm{~nm})$.

\subsection{Detection of DNA Lesions}

DNA breaks were measured in DNA of mechanically isolated nuclei by a single cell gel electrophoresis (comet) assay [17]. Nuclear suspension from approximately $100 \mathrm{mg}$ of frozen plant samples was dispersed in melted 0.7\% low melting agarose cat. 15510-027 (Life Technologies sro., Prague, Czech Republic) at $40{ }^{\circ} \mathrm{C}$ and four aliquots were immediately pipetted in duplicate on two agarose-coated microscope slides, covered with a cover slips and chilled on ice for $1 \mathrm{~min}$ to let agarose solidify. After removal of cover slips, slides were immersed in lysis solution $(2.5 \mathrm{M} \mathrm{NaCl}, 10 \mathrm{mM}$ Tris-HCl, $0.1 \mathrm{M}$ EDTA, and 1\% sodium N-lauroylsarcosinate, $\mathrm{pH} 7.6$ ) at room temperature for $1 \mathrm{~h}$ to dissolve cellular membranes and remove attached proteins. The whole procedure from chopping plant biomass by razor blade to placement into lysis solution took approximately $3 \mathrm{~min}$.

After lysis, slides were either first incubated for $20 \mathrm{~min}$ in unwinding solution $(0.3 \mathrm{M} \mathrm{NaOH}$, $5 \mathrm{mM}$ EDTA, pH 13.5) to partially unwind the DNA double helix and reveal single strand breaks (SSBs) (A/N protocol) $[17,18]$, or, for detection of double strand breaks (DSBs), were directly processed without unwinding (N/N protocol), twice equilibrated for $5 \mathrm{~min}$ in tris-acetate (TA) electrophoresis buffer (100 mM Tris, $300 \mathrm{mM}$ sodium acetate, $\mathrm{pH} 9)$ to remove salts and detergents [19,20]. After $3 \mathrm{~min}$ of electrophoresis at $1 \mathrm{~V} / \mathrm{cm}$, slides were washed in $70 \%$ and $100 \%$ ethanol and air dried. UV dimers were detected as SSBs induced at the sites of dimers by digestion with CPD specific T4 endonuclease $\mathrm{V}$ (T4EndoV) prior to alkaline unwinding in the $\mathrm{A} / \mathrm{N}$ protocol. T4EndoV was prepared as a crude lysate of an overexpressing bacterial strain [21] and applied as described in Holá et al., 2015 [11].

Comets were stained with SYBR Gold (Molecular Probes/Invitrogen), viewed in epifluorescence with a Nikon Eclipse 800 microscope (Nikon CEE GmbH, Prague, Czech Republic) and blind-evaluated by the LUCIA Comet Assay (LIM Inc., Prague, Czech Republic). The fraction of DNA in comet tails 
(\% T DNA) was used as a measure of DNA damage. The percentage of remaining damage (\% DSB remaining) after a given repair time $\left(t_{x}\right)$ is defined as:

$$
\% \text { damage remaining }(\mathrm{tx})=\frac{\text { mean } \% \mathrm{~T} \text { DNA }(\mathrm{t} x)-\text { mean } \% \mathrm{~T} \text { DNA }(\text { control })}{\text { mean } \% \mathrm{~T} \text { DNA }(\mathrm{t} 0)-\text { mean } \% \mathrm{~T} \text { DNA }(\text { control })} \times 100
$$

Data in this study were obtained from at least three independent experiments, in which 25 comets were evaluated in each of four independent gels providing at least 300 comets analyzed per experimental point. Repair kinetics was analyzed by Prism v.5 program (GrafPad Software Inc., La Jolla, CA, USA).

\section{Results}

\subsection{Preparation of Streptophyta for Genotoxicity Testing}

To obtain comparable conditions for algal strains Klebsormidium and Zygnema and moss P. patens, we carried out all experiments in liquid culture, which was left to recover for 1 day after mechanical shearing. According to previous experience with Physcomitrella [12], the use of short fragments avoids differences due to growth stage and history of culture. In regenerating moss filaments of 2-7 cells length, are up to $50 \%$ of the cells potentially mitotically active apical cells, what is in number close to number of cells in algal fragments where all cells have potency to divide. Outcome of shearing is illustrated on Figure 1 and is clearly similarly effective in all strains.

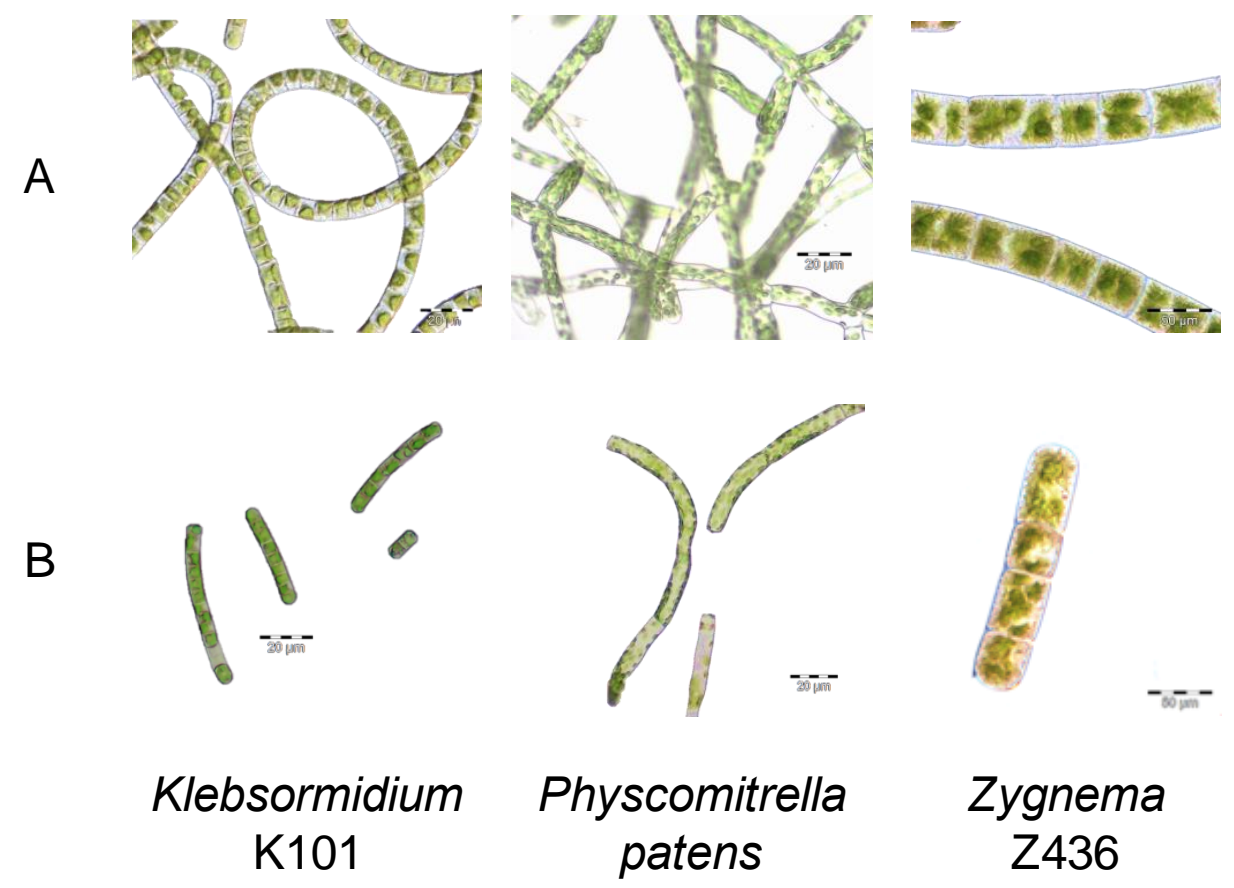

Figure 1. Fragmentation of the algae Klebsormidium flaccidum K10, Zygnema sp. Z436 and of the moss Physcomitrella patens. Regularly growing algae and moss on plates (A) were sheared to smaller fragments of app. 2-7 cells in length (B).

\subsection{Sensitivity of Klebsormidium and Zygnema to Genotoxic Treatment}

The recovery of the algae K. flaccidum K101 and Zygnema sp. Z436 treated for 30 min with different concentrations of bleomycin and MMS was monitored as growth of spot inocula over a 2-week period (Figure 2). Algal growth was unimpaired by bleomycin treatment at concentrations of $30 \mu \mathrm{g} / \mathrm{mL}$ in K. flaccidum $\mathrm{K} 101$ or $10 \mu \mathrm{g} / \mathrm{mL}$ in Zygnema sp. Z436. At higher concentrations (100-300 $\mu \mathrm{g}$ bleomycin/mL), the growth of K. flaccidum K101 was inhibited, whilst for Zygnema sp. Z436 the 
concentration $30 \mu \mathrm{g}$ bleomycin $/ \mathrm{mL}$ was already severely detrimental and at $100 \mu \mathrm{g} / \mathrm{mL}$ was almost lethal. This indicates nearly one order of magnitude higher sensitivity of Zygnema sp. Z436 to destruction by bleomycin. Nevertheless, few surviving filaments (enlarged sections in Figure 2C) are still present in the foci of otherwise 'dead' cells. Similarly, a higher sensitivity of Zygnema sp. Z436 to MMS than K. flaccidum K101 is displayed in Figure 2B,D.

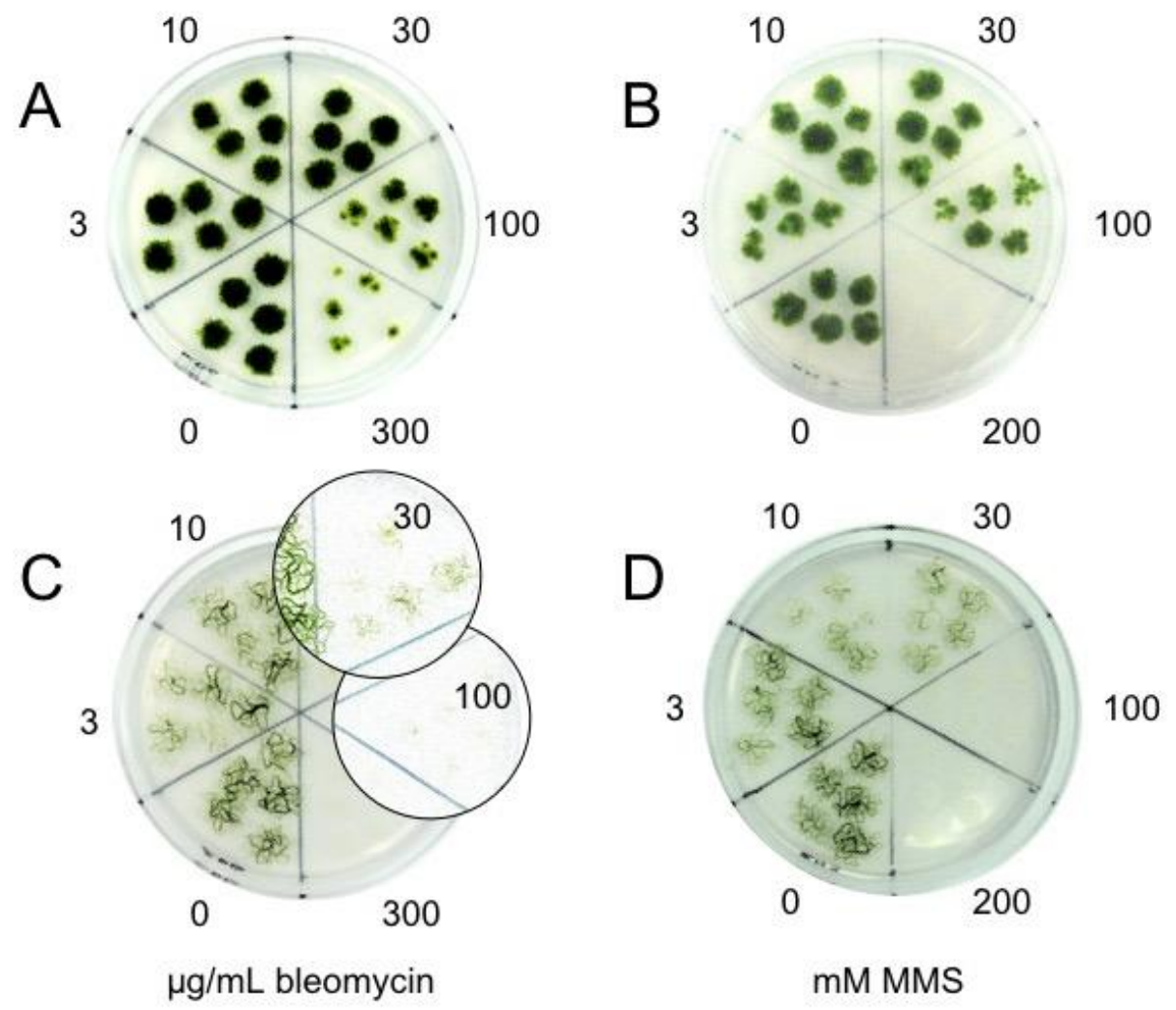

Figure 2. Sensitivity of the K. flaccidum K101 (A,B) and Zygnema sp. Z436 (C,D) to bleomycin $(\mathbf{A}, \mathbf{C})$ and methyl methanesulfonate (MMS) $(\mathbf{B}, \mathbf{D})$ treatment. The cultures of fragmented algae were treated for $30 \mathrm{~min}$ in liquid bold-basal medium (BBM) with the indicated concentrations of bleomycin and MMS, then spot inoculated on BBM agar plates and grown under continuous light for 2 weeks. Few filaments of Zygnema sp. Z436 survived or developed from surviving cells treated with 30 and $100 \mu \mathrm{g}$ bleomycin/mL. To visualize surviving individual filaments the enlarged sections with increased contrast are inserted in panel C. No viable cells were found at $300 \mu \mathrm{g}$ bleomycin $/ \mathrm{mL}$, as well as at 100 and 200 mM MMS. Less sensitive Klebsormidium K101did not survive only the highest, $200 \mathrm{mM}$ MMS treatment.

The morphology of cells of K. flaccidum K101 (Figure 3A) and Zygnema sp. Z436 (Figure 3B) also differed in response to bleomycin and MMS treatment. No apparent damage of algal cells was observed one day after genotoxin treatment, including the highest concentrations, except visible shrinkage of chloroplasts in some Zygnema cells treated with $100 \mathrm{mM}$ MMS. During following 7 days of cultivation no recovery from cellular damage was observed and all cells in filaments exhibited irreversible morphological changes such as disintegration of organelles, especially of chloroplast and chlorophyll degradation. Less cell damage was observed in Zygnema sp. Z436 after bleomycin treatment, often with presence of damaged and not damaged cells in one filament. By contrast to Zygnema fewer morphological changes were seen in K. flaccidum K 101 cells following bleomycin and MMS treatments.

The sensitivity of $P$. patens to bleomycin treatment and UV irradiation has been previously described [12]. 




Figure 3. Effect of genotoxic treatment on morphology of algal cells. The suspension cultures of K. flaccidum K101 (A) and Zygnema sp. Z436 (B) in bold basal medium (BBM) were treated 30 min with $100 \mu \mathrm{g}$ bleomycin/mL or $100 \mathrm{mM}$ MMS, followed by 7 days recovery cultivation in BBM medium under continuous light. No morphological changes are visible in Klebsormidium K101 cells first day after bleomycin or MMS treatment depicted as treated cells. Certain tendency to chlorophyll bleaching could be seen in MMS treated samples of Klebsormidium K101 cells after 7 days of recovery. Irreversible damage of Zygnema sp. Z436 cells due to disintegration of organelles and chlorophyll degradation is manifested after 7 days recovery from MMS treatment.

\subsection{DNA Damage in Klebsormidium, Zygnema and Physcomitrella}

To characterize differences in vulnerability to DNA damage between algae and moss, we used comet assay and treatments with bleomycin to study induction and repair of DSBs, MMS to study induction and repair of SSBs and UVC irradiation to study induction and dark repair of CPDs (Figure 4). Bleomycin and MMS treatment induces several times more DNA lesions in Klebsormidium K101 and Physcomitrella than in Zygnema Z436 (Figure 4A,B).

To rule out distinct responses to genotoxic treatment unique to the K. flaccidum K101 and Zygnema sp. Z436 strains, we compared the sensitivity of three strains of each genera to induction of DSBs. We tested two strains of K. flaccidum denoted K101 and K293, and K. elegans K292. Zygnematales were represented by two strains of Zygnema sp. Z436 and Z294, and by Z. circumcarinatum Z181. The sensitivity of Physcomitrella to bleomycin induction of DSBs is similar to that of Klebsormidium strains (Figure 5). 
Physcomitrella and Klebsormidium share a similar vulnerability and response of cells to genotoxin treatment in contrast to distinct Zygnema. Contrary to bleomycin and MMS treatment Zygnema sp. Z436, and particularly Physcomitrella, are far more sensitive to the induction of CPDs than K. flaccidum K101 (Figure 4C).

A

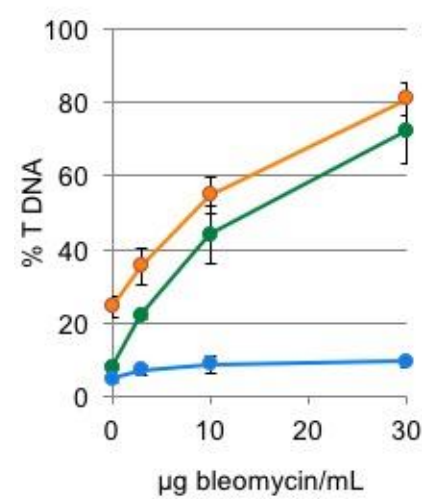

B

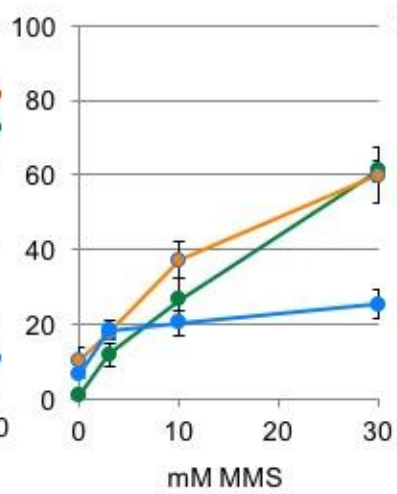

C

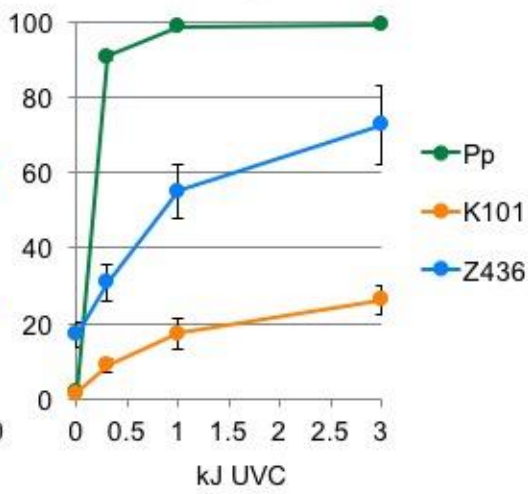

Figure 4. The dose-responses of algae and moss to genotoxic treatment. The suspension cultures of K. flaccidum K101, Zygnema sp. Z436 and Physcomitrella (Pp)were treated with 0-30 $\mu \mathrm{g} / \mathrm{mL}$ bleomycin (A) and 0-30 mM MMS for $1 \mathrm{~h}$ (B). The same cultures spotted on agar plates were irradiated with 0-3 kJ UVC (C). Upon treatment, the culture was blotted dry and immediately frozen in liquid nitrogen prior extraction of nuclei and analysis of DNA damage by the comet assay. The extent of DNA damage is indicated by the proportion of fragmented DNA in the 'comet tails' (\% T DNA). Double strand breaks (DSBs) were followed as an end point of bleomycin treatment, single strand breaks (SSBs) as an endpoint of MMS treatment and DNA pyrimidine (CPDs) as an endpoint of UVC irradiation detected as T4EndoV sensitive sites.

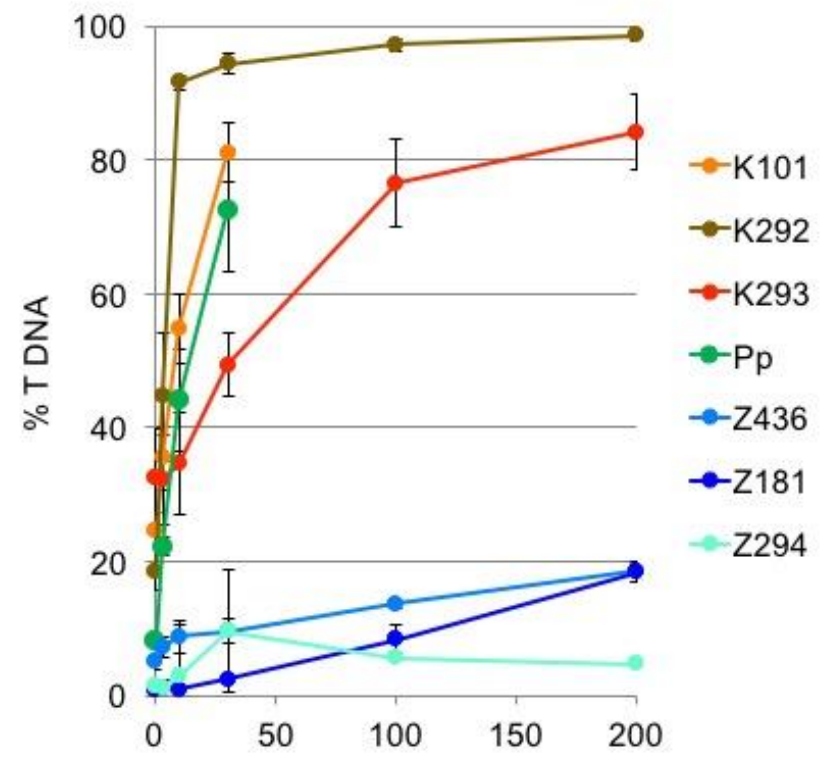

$\mu \mathrm{g}$ bleomycin $/ \mathrm{mL}$

Figure 5. Bleomycin dose-response of Klebsormidium and Zygnema species. The cultures of K. flaccidum K101 (heavy orange), K. elegans K292 (brown), K. flaccidum K293 (red), Zygnema sp. Z436 (mid blue), Z. circumcarinatum Z181 (dark blue), Zygnema sp. Z294 (cyan), and Physcomitrella (Pp, green) were treated with an extended concentration range of bleomycin $(0-200 \mu \mathrm{g} / \mathrm{mL})$ for $1 \mathrm{~h}$ and analyzed for induction of DSBs. 


\subsection{Double Strand Break Repair}

Repair kinetics was studied in 1-day regenerating cultures (Figure 6). The repair of DSBs induced by $30 \mu \mathrm{g}$ bleomycin/mL could be approximated by two-phase decay kinetics in Klebsormidium K101, Zygnema sp. Z436 and Physcomitrella strains. The observed half-life of DSBs during the first, rapid phase of the repair is $4.1 \mathrm{~min}$. in Physcomitrella and $5.8 \mathrm{~min}$ in K. flaccidum K101, whilst only 1.9 min in Zygnema sp. Z436. This indicates that while K. flaccidum K101 and Physcomitrella repair DSBs at approximately same rate, Zygnema sp. Z436 repairs DSBs more than twice as fast.

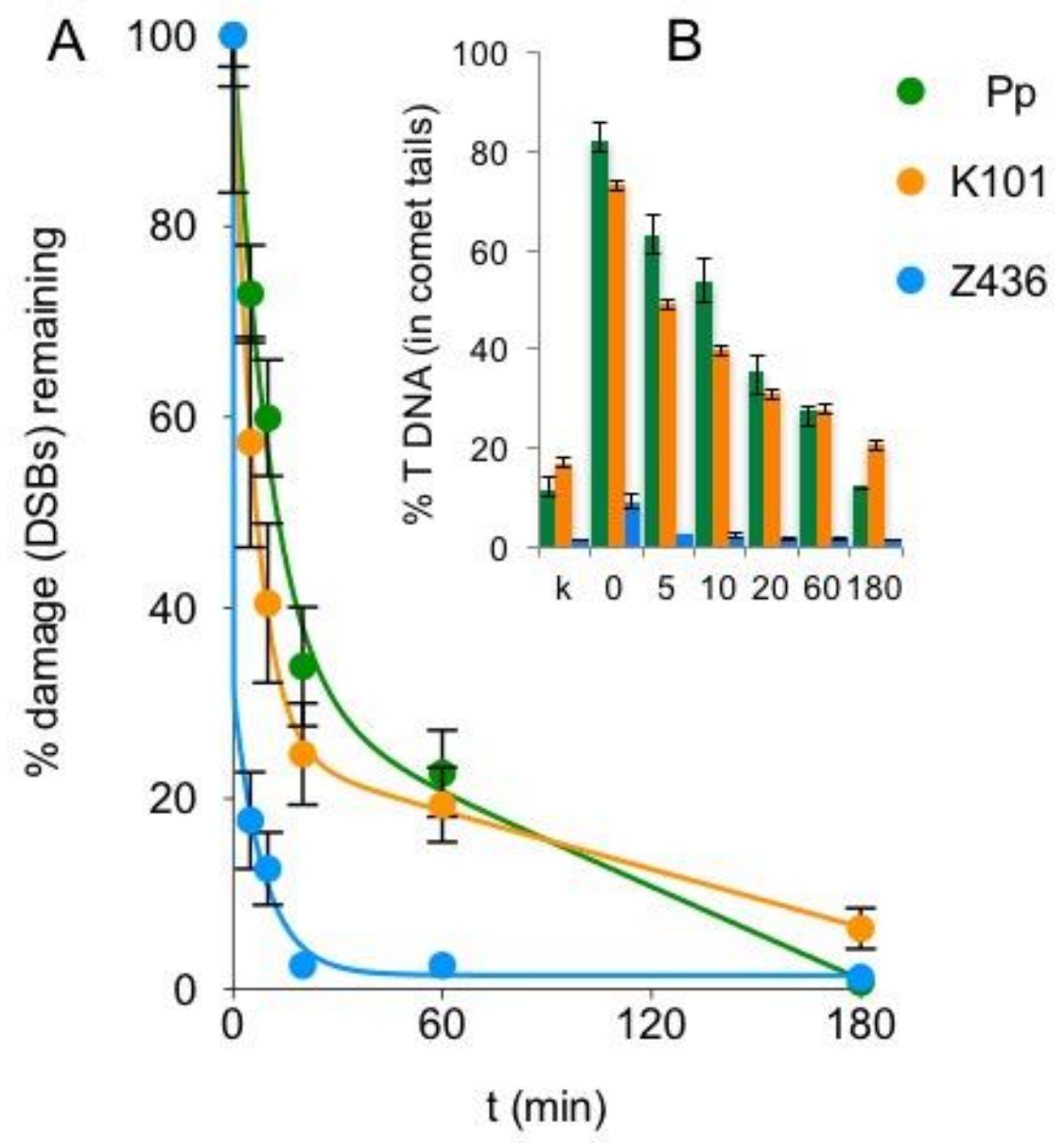

Figure 6. Kinetics of DSBs repair. The cultures of Klebsormidium flaccidum K101 (orange), Zygnema sp Z436 (blue) and Physcomitrella (green) were treated for $1 \mathrm{~h}$ with $30 \mu \mathrm{g}$ bleomycin $/ \mathrm{mL}$ and allowed to recover for the indicated times. The fraction of DNA measured in comet tails \% T DNA (embedded graph B) was normalized to $100 \%$ damage after treatment $(t=0)$, to highlight differences among each strain. The graph plot A was generated as approximation of two-phase decay kinetics through the experimental points by the Prism v.5 program.

\section{Discussion}

\subsection{Response to Genotoxic Treatment}

Transition from water to land and changes from wet to arid environments challenge these species through the accompanying salt and osmotic stress and exposure to environmental inorganic salts, heavy metals and chemicals, some of which are currently widespread as man-made pollutants. In parallel they are also exposed to such physical stresses as extreme temperature fluctuations and exposure to IR and UV radiation. All these factors cause direct damage of DNA and impair cellular functions and this is why plants consequentially evolved multiple mechanisms to avoid, tolerate, or repair DNA damage. 
In this study, we focused on the immediate responses of plants. To provide uniform conditions for the genotoxic testing of the selected streptophyte species, we used culture of small fragments. The use of small vs. long fragments was devised to study kinetic of DSB repair in apical versus differentiated cells of Physcomitrella protonema [12]. In this study, we used the same approach for Klebsormidium and Zygnema because their algal filament morphology is similar moss protonemata. Of course, there is an obvious difference in polar growth of moss protonema with possible branching of internal cells in contrast to algal filaments that retain the undifferentiated capacity of every cell within filament to divide. In the small fragments the differences in numbers of potentially dividing cells are limited and comparable at the start of experiment. Nevertheless, these conditions represent an experimental approximation and might not necessarily fully reflect natural conditions [22].

As is evident from Figure 1, the cell size of Physcomitrella and Klebsormidium is closer than that of Zygnema. The possible explanation of this structural difference might arise from their natural habitats and evolutionarily developed tolerance to desiccation. The abundance and specific localization of callose correlates with the higher desiccation tolerance of Klebsormidium when compared with Zygnema [23]. The distribution of callose within the cells provides an explanation for the frequent occurrence of Klebsormidium strains or species in hot and cold deserts, which are characterized by low water availability and other stressful conditions [24], whereas Zygnema is better adapted to Arctic and Antarctic desiccation. The state of recovery from genotoxic stress after 7 days (Figure 3) shares similarities with desiccation stress [23,25-27]. Morphological changes occur over time and do not revert, indicating the induction of permanent changes. This process takes several days and in Zygnema, a senescent phenotype rapidly leads to necrosis in all cells. After an acute (30 min) exposure to genotoxic treatment, algae do not form akinete or pre-akinete cells as they do following desiccation stress indicating a terminal fate without possible recovery. Pre-akinetes are crucial for the aero-terrestrial lifestyle of Zygnema $[25,28]$. The hypersensitive cell morphology phenotype is more strongly manifested in Zygnema sp. Z436 than in K. flaccidum K101 after bleomycin treatment. This observation is also confirmed by growth tests of inocula of treated algal cultures, where K. flaccidum K101, but not Zygnema sp. Z436 survives on plates despite treatment with 3-fold higher concentrations 300 vs. $100 \mu \mathrm{g} / \mathrm{mL}$ of bleomycin and 100 vs. $30 \mathrm{mM}$ MMS, respectively (Figure 2).

\subsection{DNA Damage and Repair}

In contrast to the higher sensitivity of Zygnema Z436 over Klebsormidium K101 in growth tests (Figure 2), Zygnema Z436 shows far less vulnerability to induction of DSBs by bleomycin and of SSBs by MMS treatment (Figure 4). As depicted in Figure 5, different sensitivities to genotoxic treatment are not solely associated with the K. flaccidum K101 and Zygnema sp. Z436 strains, but are typical for the whole Klebsormidium and Zygnema groups. Additionally, repair rates of induced DSBs are 2- to 3-fold faster in Zygnema than in Klebsormidium and Physcomitrella.

Choi et al. [1] recently described an effect of $\gamma$-irradiation of Arctic Zygnema with doses up to $5 \mathrm{kGy}$ and found that the photosynthetic efficiency markedly decreased, whereas antioxidant capacity significantly increased. Proteomic analysis using 2D electrophoresis combined with mass spectrometry allowed reliable identification of upregulated proteins by peptide fingerprinting. Identified among the upregulated proteins were DNA ligase and ATP-dependent DNA helicase (KU80 subunit), both related to DSBs repair by Non-Homologous End Joining (NHEJ) pathway, and this is why authors concluded that upregulation of DNA repair-related proteins may be an underlying mechanism of radioresistance against oxidative stress caused by ionizing radiation.

Furthermore, upon desiccation Zygnema showed also an induction of stress protection mechanisms such as ROS scavenging and DNA repair. DNA damage, which is linked to ROS formation, was counteracted by a strong upregulation of repair enzymes. For example, plant orthologue of Nijmegen breakage syndrome gene (NBS1), which is a part of MRE11-RAD50-NBS1 (MRN) complex involved in recognition and repair of DSBs, was highly induced with 9.7-fold change, suggesting a higher risk of DNA damages associated with desiccation. Moreover, a number of other stress 
related molecules are produced, that is, chaperones such as late embryogenesis abundant (LEA) proteins, proteins involved in ROS scavenging and DNA repair proteins [27]. Contrary to Zygnema in Klebsormidium desiccation induced down-regulation of transcripts and complex regulation patterns. Pathways, which were strongly down-regulated, were mainly involved in integrative cellular functions, such as cell division and DNA repair mechanisms [26]. This suggests that at least from a transcriptomic point of view Zygnema and Klebsormidium have differently controlled stress response.

Direct measurement of damage in genomic DNA and its repair is a good estimate how particular cells are able to convert a non-instructional to an instructional genetic template and thus proceed through the cell cycle. Lesions induced in DNA lead either directly to DNA inactivation as a template or indirectly by increased mutagenesis due to error-prone repair in numerous genes critical for normal development, which are then manifested as a hypersensitive phenotype.

In the first alternative, DNA replication machines of algal or moss cells are challenged by lesions induced within DNA. It can be speculated that drugs targeting DNA induce more DNA lesions in dividing cells because the replicating DNA expose to the drugs in a higher chance than the tightly packed DNA in cells not dividing. The less accumulation of DNA lesions in Zygnema cells may due to the immediate death induced by drug, that is, Zygnema cells could not repair the strand-breaks, and the replication machine is sensitive to strand-breaks. It stalls the DNA replications and finally leads to cell death. The DNA replication complex in Klebsormidium and Physcomitrella cells might less sensitive to the drugs so the DNA replication and cell division are not inhibited by the drugs. Since'the DNA keeps replicating, strand-breaks accumulate in cells and present an apparent high percentage of damaged DNA.

In the second alternative, DNA lesions are repaired, and the intact template restored, but at the price of alteration of genetic information, leading to accumulation of deleterious mutations incompatible with normal growth and development. Individual cells of an algal filament acquire a different spectrum of DNA lesions and mutations during their error-prone repair, and while the majority of cells ultimately undergo necrosis and die, any surviving cell with no or only tolerated mutation in vital genes could regenerate and perpetuate. This process might account for seldom-occurring surviving cells in foci of Zygnema inocula treated with high concentration of bleomycin (insert in Figure 2).

We have previously described this phenomenon in various DNA repair mutants of Physcomitrella, where sensitive phenotype was an outcome of increased mutagenesis due to alternative error-prone repair rather than defect of repair itself [10-12]. Here it should be noted that in Zygnema the upregulated NHEJ pathway by $\gamma$-irradiation [1] is a notoriously error-prone repair pathway.

Nevertheless, it is difficult to reconcile observed differences because as we suggested above, it may reflect different routes that evolved to offset stress conditions-drought as well as genotoxic exposure. This is why the different capabilities of Klebsormidium and Physcomitrella vs. Zygnema that evolved due to varied habitat conditions might represent, on a molecular level, the evolution of different induction patterns and utilization of repair pathways in response to genotoxic stress.

\subsection{Response to UVC}

UVC induces CPDs with the same efficiency as terrestrially more relevant UVB, which can be effectively monitored as SSBs by the comet assay following digestion with the CPD specific T4EndoV. When compared to bleomycin and MMS treatment, Zygnema Z436, Klebsormidium K101 and Physcomitrella differed in their order of sensitivity to UVC (Figure 4). The reason might be that all three organisms adapted to different habitats with different need for protection against high UV irradiance and desiccation. Regular exposure of aero-terrestrial Klebsormidium species to stress factors need more effective adaptation mechanisms than hydro-terrestrial Zygnema species, which are exposed to strong UV occasionally, such as during dry season after desiccation of water bodies. 
Unlike Klebsormidium species, which have to cope daily with rapid changes of UV exposure in a vegetative state, Arctic and Antarctic Zygnema species need to form pre-akinetes, hardened by slow desiccation, to be able tolerate severe ecological stresses including UV irradiation [7]. It is difficult to find an explanation on the DNA level, nevertheless, our results are consistent with recently published results of the high tolerance of various Klebsormidium species to UV radiation determined as optimum quantum yield of photosystem II (PSII) as well as the capability to synthesize and accumulate mycosporine-like amino acids (MAAs) as 'sunscreens' to enable their aero-terrestrial lifestyle [29]. As we tested only the induction of CPDs, the most likely explanation is different protection by UV-shielding, for example due to the presence of phenolic compounds, as has been reported in Zygnema [3]. In contrast, the moss P. patens usually occupies and survives in shaded areas and this might be why it did not develop efficient passive photo-protection and thus suffers massive induction of DNA damage. We have recently reported high rate of mutagenesis in Physcomitrella due to efficient error-prone bypass of photo-dimers leading to $\mathrm{C} \rightarrow \mathrm{T}$ transitions [11]. Whether this mechanism is relevant also in Zygnema has to be established.

\section{Conclusions}

We characterized the responses of the green algae Klebsormidium and Zygnema and of the moss P. patens to induction of DSBs by bleomycin, to DNA base alkylation by MMS and to formation of CPDs after UV irradiation. These genotoxic agents with well-characterized modes of action were used to probe the vulnerability of these species to the induction and removal of DNA damage. The Klebsormidium species and Physcomitrella show a similar sensitivity toward the induction of DNA lesions and of DSB repair. In contrast Zygnema species are less sensitive to the induction of DNA damage and exercise a high rate of DSB repair. Nevertheless, contrary to fewer lesions in DNA, Zygnema is more sensitive to genotoxic treatment than Klebsormidium and Physcomitrella.

Acknowledgments: This work was supported by the Czech Science Foundation (16-01137S) and by the Ministry of Education, Youth and Sports of the Czech Republic under the project CEITEC 2020 (LQ1601) and COST Action CA15132 (grant LTC17047/2017). AL would like to thank Romana Bícová and Lenka Štenclová (Institute of Soil Biology) for algal cultivation and technical help.

Author Contributions: K.J.A. and J.Fa. conceived and designed the experiments; R.V., A.L., M.L., P.R. and M.H. performed the experiments; K.J.A. and J.Fu. analyzed the data; K.J.A., A.L., J.Fu. and J.Fa. wrote the paper.

Conflicts of Interest: The authors declare no conflict of interest.

\section{References}

1. Choi, J.-I.; Yoon, M.; Lim, S.; Kim, G.H.; Park, H. Effect of $\gamma$-irradiation on physiological and proteomic changes of Arctic Zygnema sp. (Chlorophyta, Zygnematales). Phycologia 2015, 54, 9. [CrossRef]

2. Pichrtova, M.; Hajek, T.; Elster, J. Osmotic stress and recovery in field populations of Zygnema sp. (Zygnematophyceae, Streptophyta) on Svalbard (High Arctic) subjected to natural desiccation. FEMS Microbiol. Ecol. 2014, 89, 270-280. [CrossRef] [PubMed]

3. Pichrtova, M.; Remias, D.; Lewis, L.A.; Holzinger, A. Changes in phenolic compounds and cellular ultrastructure of arctic and antarctic strains of Zygnema (Zygnematophyceae, Streptophyta) after exposure to experimentally enhanced uv to par ratio. Microb. Ecol. 2013, 65, 68-83. [CrossRef] [PubMed]

4. Kaplan, F.; Lewis, L.A.; Herburger, K.; Holzinger, A. Osmotic stress in Arctic and Antarctic strains of the green alga Zygnema (Zygnematales, Streptophyta): Effects on photosynthesis and ultrastructure. Micron 2013, 44, 317-330. [CrossRef] [PubMed]

5. Rindi, F.; Guiry, M.D.; Lopez-Bautista, J.M. Distribution, morphology, and phylogeny of Klebsormidium (Klebsormidiales, Charophyceae) in urban environments in Europe. J. Phycol. 2008, 44, 1529-1540. [CrossRef] [PubMed]

6. Mikhailyuk, T.; Glaser, K.; Holzinger, A.; Karsten, U. Biodiversity of (Streptophyta) from alpine biological soil crusts (Alps, Tyrol, Austria, and Italy). J. Phycol. 2015, 51, 750-767. [CrossRef] [PubMed] 
7. Pichrtova, M.; Kulichova, J.; Holzinger, A. Nitrogen limitation and slow drying induce desiccation tolerance in conjugating green algae (Zygnematophyceae, Streptophyta) from polar habitats. PLoS ONE 2014, 9, e113137. [CrossRef] [PubMed]

8. Cove, D.J.; Knight, C.D.; Lamparter, T. Mosses as model systems. Trends Plant Sci. 1997, 2, 99-105. [CrossRef]

9. Zimmer, A.; Lang, D.; Richardt, S.; Frank, W.; Reski, R.; Rensing, S.A. Dating the early evolution of plants: Detection and molecular clock analyses of orthologs. Mol. Genet. Genom. 2007, 278, 393-402. [CrossRef] [PubMed]

10. Hola, M.; Kozak, J.; Vagnerova, R.; Angelis, K.J. Genotoxin induced mutagenesis in the model plant Physcomitrella patens. Biomed Res. Int. 2013, 2013, 535049. [CrossRef] [PubMed]

11. Holá, M.; Vagnerova, R.; Angelis, K.J. Mutagenesis during plant responses to UVB radiation. Plant Physiol. Biochem. 2015, 93, 29-33. [CrossRef] [PubMed]

12. Kamisugi, Y.; Schaefer, D.G.; Kozak, J.; Charlot, F.; Vrielynck, N.; Holá, M.; Angelis, K.J.; Cuming, A.C.; Nogue, F. MRE11 and RAD50, but not NBS1, are essential for gene targeting in the moss Physcomitrella patens. Nucleic Acids Res. 2012, 40, 3496-3510. [CrossRef] [PubMed]

13. Steighner, R.J.; Povirk, L.F. Bleomycin-induced DNA lesions at mutational hot spots: Implications for the mechanism of double-strand cleavage. Proc. Natl. Acad. Sci. USA 1990, 87, 8350-8354. [CrossRef] [PubMed]

14. Borderie, F.; Tete, N.; Cailhol, D.; Alaoui-Sehmer, L.; Bousta, F.; Rieffel, D.; Aleya, L.; Alaoui-Sosse, B. Factors driving epilithic algal colonization in show caves and new insights into combating biofilm development with UV-C treatments. Sci. Total Environ. 2014, 484, 43-52. [CrossRef] [PubMed]

15. Bischoff, H.W.; Bold, H.C. Phycological studies IV. In Some Soil Algae from Enchanted Rock and Related Algal Species; University of Texas: Austin, TX, USA, 1963; pp. 1-95.

16. Knight, C.D.; Cove, D.J.; Cuming, A.C.; Quatrano, R.S. Moss gene technology. In Molecular Plant Biology; Gilmartin, P.M., Bower, C., Eds.; Oxford University Press: Oxford, UK, 2002; Volume 2, pp. 285-299.

17. Angelis, K.J.; Dusinska, M.; Collins, A.R. Single cell gel electrophoresis: Detection of DNA damage at different levels of sensitivity. Electrophoresis 1999, 20, 2133-2138. [CrossRef]

18. Menke, M.; Chen, I.; Angelis, K.J.; Schubert, I. DNA damage and repair in Arabidopsis thaliana as measured by the comet assay after treatment with different classes of genotoxins. Mutat. Res. 2001, 493, 87-93. [CrossRef]

19. Kozak, J.; West, C.E.; White, C.; da Costa-Nunes, J.A.; Angelis, K.J. Rapid repair of DNA double strand breaks in Arabidopsis thaliana is dependent on proteins involved in chromosome structure maintenance. DNA Repair (Amst) 2009, 8, 413-419. [CrossRef] [PubMed]

20. Olive, P.L.; Banath, J.P. The comet assay: A method to measure DNA damage in individual cells. Nat. Protoc. 2006, 1, 23-29. [CrossRef] [PubMed]

21. Collins, A.R. The use of bacterial repair endonucleases in the comet assay. Methods Mol. Biol. 2011, 691, 137-147. [PubMed]

22. Kovalchuk, I.; Molinier, J.; Yao, Y.; Arkhipov, A.; Kovalchuk, O. Transcriptome analysis reveals fundamental differences in plant response to acute and chronic exposure to ionizing radiation. Mutat. Res. 2007, 624, 101-113. [CrossRef] [PubMed]

23. Herburger, K.; Holzinger, A. Localization and quantification of callose in the streptophyte green algae Zygnema and Klebsormidium: Correlation with desiccation tolerance. Plant Cell Physiol. 2015, 56, 2259-2270. [PubMed]

24. Karsten, U.; Herburger, K.; Holzinger, A. Living in biological soil crust communities of african deserts-physiological traits of green algal Klebsormidium species (Streptophyta) to cope with desiccation, light and temperature gradients. J. Plant Physiol. 2016, 194, 2-12. [CrossRef] [PubMed]

25. Holzinger, A.; Pichrtova, M. Abiotic stress tolerance of charophyte green algae: New challenges for omics techniques. Front. Plant Sci. 2016, 7, 678. [CrossRef] [PubMed]

26. Holzinger, A.; Kaplan, F.; Blaas, K.; Zechmann, B.; Komsic-Buchmann, K.; Becker, B. Transcriptomics of desiccation tolerance in the streptophyte green alga Klebsormidium reveal a land plant-like defense reaction. PLoS ONE 2014, 9, e110630. [CrossRef] [PubMed]

27. Rippin, M.; Becker, B.; Holzinger, A. Enhanced desiccation tolerance in mature cultures of the streptophytic green alga Zygnema circumcarinatum revealed by transcriptomics. Plant Cell Physiol. 2017. [CrossRef] [PubMed] 
28. Herburger, K.; Lewis, L.A.; Holzinger, A. Photosynthetic efficiency, desiccation tolerance and ultrastructure in two phylogenetically distinct strains of alpine Zygnema sp. (Zygnematophyceae, Streptophyta): Role of pre-akinete formation. Protoplasma 2015, 252, 571-589. [CrossRef] [PubMed]

29. Kitzing, C.; Karsten, U. Effects of UV radiation on optimum quantum yield and sunscreen contents in members of the genera Interfilum, Klebsormidium, Hormidiella and Entransia (Klebsormidiophyceae, Streptophyta). Eur. J. Phycol. 2015, 50, 9. [CrossRef]

(C) 2017 by the authors. Licensee MDPI, Basel, Switzerland. This article is an open access article distributed under the terms and conditions of the Creative Commons Attribution (CC BY) license (http:/ / creativecommons.org/licenses/by/4.0/). 